\title{
Catalyst-Free Growth of Magnesium Oxide Whiskers and Their Characteristics
}

\author{
H.W. KIM ${ }^{a, *}$, M.H. KONG ${ }^{a}$ AND J.-H. YANG ${ }^{b}$ \\ ${ }^{a}$ Division of Materials Science and Engineering, Inha University \\ Incheon 402-751, Republic of Korea \\ ${ }^{b}$ Advanced Materials R\&D Center, Korea Institute of Industrial Technology \\ Republic of Korea
}

\begin{abstract}
This study reports the production of magnesium oxide $(\mathrm{MgO})$ whiskers on silicon $(\mathrm{Si})$ substrates by the thermal heating of $\mathrm{MgB}_{2}$ powders. We investigated the structural properties of the as-synthesized whiskers by using $\mathrm{X}$-ray diffraction, transmission electron microscopy, selected area electron diffraction, and scanning electron microscopy. The product consisted of onedimensional whiskers with a square cross-section. The whiskers had a singlecrystalline cubic structure of $\mathrm{MgO}$. The photoluminescence measurement with the Gaussian fitting exhibited visible light emission bands centered at $2.39 \mathrm{eV}$ and $2.91 \mathrm{eV}$. We proposed the growth of $\mathrm{MgO}$ whiskers to follow the vapor-solid mechanism.
\end{abstract}

PACS numbers: 81.07.-b, 78.55.-m

\section{Introduction}

Low-dimensional materials such as one-dimensional (1D) nanoscale or submicron scale materials are currently being exploited as active components in a wide range of technological applications in various fields, due to their high surface-tovolume ratio and better material characteristics. Magnesium oxide $(\mathrm{MgO})$ is a typical wide-band-gap insulator. It has found many important applications for use in catalysis, refractory material industry, paint, and superconductors. Furthermore, $1 \mathrm{D} \mathrm{MgO}$ structures can display a unique capability to pin the magnetic flux lines within a high-temperature superconductor. In the present work, we have successfully fabricated $\mathrm{MgO}$ rod-like structures on Si substrates without using metal catalysts. The successful synthesis of whiskers on the conventional $\mathrm{Si}$ substrates without using metal catalysts will pave the way for integration of future devices with developed Si integrated circuit technology.

\section{Experimental}

The synthesis process was carried out in a quartz tube. The source material was pure $\mathrm{MgB}_{2}$ powders. We employed standard polished $p$-type (100) $\mathrm{Si}$

*corresponding author; e-mail: hwkim@inha.ac.kr 
substrates. The distance between the source material-containing boat and the substrate was approximately $10 \mathrm{~mm}$. During the experiment, the furnace was maintained at a temperature of $900^{\circ} \mathrm{C}$ for $2 \mathrm{~h}$ with the ambient gas $\left(\mathrm{Ar}+\mathrm{O}_{2}\right)$ at a constant total pressure of 2 Torr. The typical percentage of $\mathrm{O}_{2}$ and Ar partial pressure, respectively, were set to approximately 2.7 and $97.3 \%$. The apparatus to analyse the products was described in this issue (see paper written by Kim et al. [1].

\section{Results and discussion}

The X-ray diffraction (XRD) pattern shown in Fig. 1 reveals the overall crystal structure of the product. The recognizable diffraction peaks correspond to the cubic MgO structure with lattice constants of $a=0.421 \mathrm{~nm}$ (JCPDS: 04-0829). No impurities, such as unreacted $\mathrm{Mg}$ or other magnesium oxides, were detected.

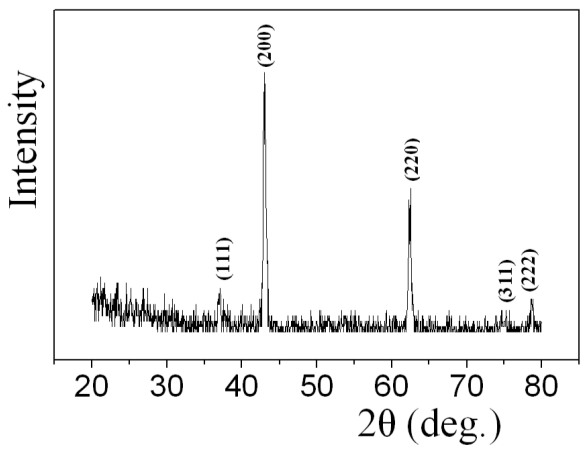

Fig. 1. (a) XRD pattern of the product.

Since the angle of the incident beam to the substrate surface was about $0.5^{\circ}$ in the present XRD measurements, we conclude that the peaks originate mainly from the product. Figure $2 \mathrm{a}$ is a side-view scanning electron microscopy (SEM) image of the product on the substrate surface, exhibiting the random growth directions of the whiskers. The whiskers have a straight-line morphology along the length direction. Figure 2b is a top-view SEM image, showing the bare-Si substrate (dark region) as well as whiskers. Statistical analysis of many SEM images shows that the whiskers have diameters ranging from 270 to $540 \mathrm{~nm}$. Close examination (Fig. 2c) reveals that the cross-section of the whiskers is close to square-shaped with no nanoparticle at their tips. We further examined the crystalline structure of the individual whisker by transmission electron microscopy (TEM). Figure 3a shows the lattice-resolved high resolution TEM (HRTEM) image taken near the side edge along a typical whisker. The interplanar spacing is about $0.21 \mathrm{~nm}$, corresponding to the $\{200\}$ plane of cubic $\mathrm{MgO}$. The selected area electron diffraction (SAED) pattern shown in the inset can be indexed for the [001] zone axis of $\mathrm{MgO}$, revealing the single-crystalline nature of the whiskers. The SAED pattern can be indexed (their $h k l$ indices are given in inset) according to their cubic $\mathrm{MgO}$ structure. 

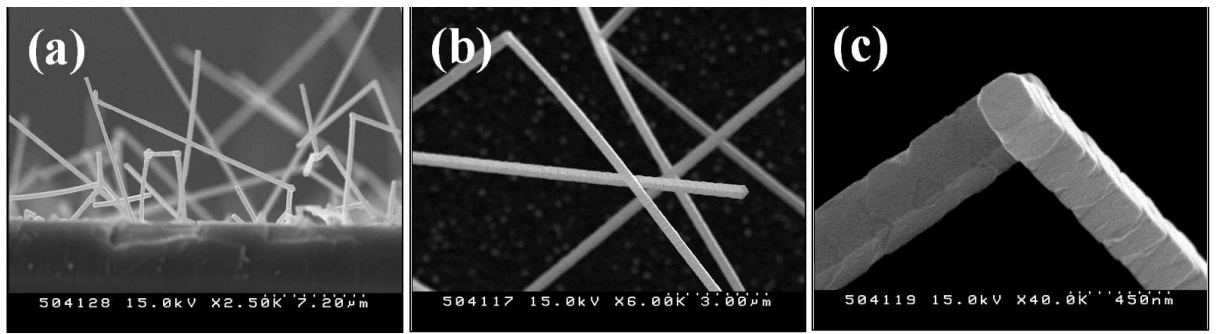

Fig. 2. (a) Side-view SEM image, (b) top-view SEM image, and (c) high magnification SEM image of the product.
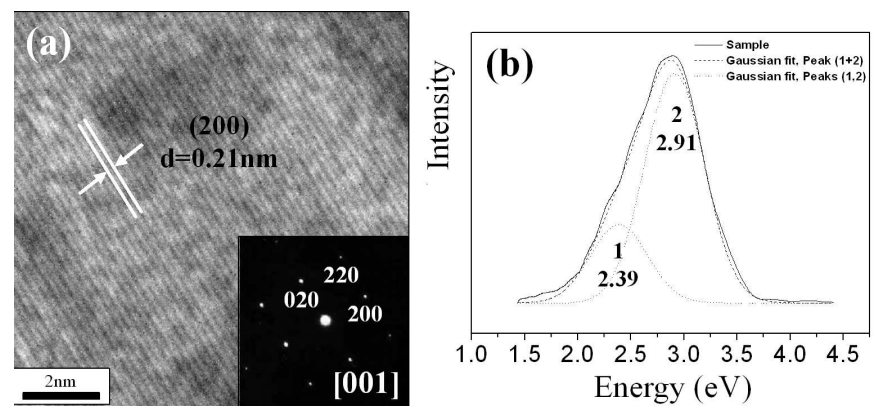

Fig. 3. (a) HRTEM image (inset: associated SAED pattern recorded along the [001] zone axis). (b) Photoluminescence spectrum from the product.

Figure 3b shows the photoluminescence (PL) spectrum measured at room temperature. After multi-peak Gaussian fitting to two major peaks of the PL spectrum, we found that the Gaussian curves fit the original curves almost perfectly. Therefore, the PL spectrum mainly consists of two bands, peaking at approximately $2.91 \mathrm{eV}$ in blue region and $2.39 \mathrm{eV}$ in blue-green region, respectively. The similar blue emission has been previously observed in PL spectra of $\mathrm{MgO}$ nanowires [2] and $\mathrm{MgO}$ nanoplates [3], whereas the blue-green emission was found from $\mathrm{MgO}$ nanobelts [4]. Both blue and blue-green emissions may originate from defects in $\mathrm{MgO}$, such as oxygen vacancies [3-5]. In the present high-temperature evaporation process, oxygen vacancies should be generated [6].

No metal catalyst was used in the present study, and no particle was observed at the ends of the whiskers in our SEM observations (Fig. 2). Therefore, the growth mechanism of the whiskers can be understood on the basis of a self-catalytic process with the characteristics of vapor-solid (VS) growth process, corresponding to a diffusion-limited process in a supersaturated environment [7]. Brief scheme on the formation of $\mathrm{MgO}$ whiskers is shown in Fig. 4. $\mathrm{Mg}$ vapor may combine with $\mathrm{O}_{2}$ gas, rapidly generating $\mathrm{MgO}$ vapor. The $\mathrm{MgO}$ vapor directly deposits on the substrate and grows into $1 \mathrm{D}$ whiskers. Two-dimensional square island nucleation is a usual phenomenon in the $\mathrm{MgO}$ film deposition process [8]. The square cross- 
section of whiskers reveals that the $\mathrm{MgO}$ whiskers have been elongated from the square nuclei (Fig. 2c). From Figs. 2a and 2b we observed that $\mathrm{MgO}$ whiskers have been generated on Si substrates, without forming the predeposited $\mathrm{MgO}$ layers. According to the VS mechanism, under low enough supersaturation in the present study, we conclude that the square islands on substrate will elongate and preferentially form the $1 \mathrm{D}$ whiskers.

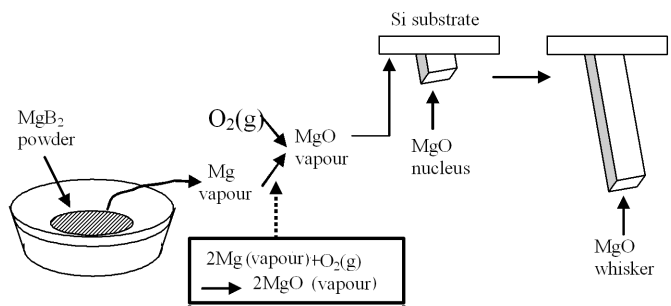

Fig. 4. Schematic illustration of $\mathrm{MgO}$ whiskers via the VS mechanism.

In conclusion, we successfully synthesized the $\mathrm{MgO}$ whiskers on bare Si substrate via a heating $\mathrm{MgB}_{2}$ powders at $900^{\circ} \mathrm{C}$. SEM images indicate that the $\mathrm{MgO}$ whiskers have average diameters in the range of $270-540 \mathrm{~nm}$. No catalytic particle is observed on the ends of the as-synthesized whiskers. XRD and energy-dispersive $\mathrm{X}$-ray (EDX) analyses coincidentally show that the $\mathrm{MgO}$ structures are of single crystalline cubic structure. A growth mechanism based on the VS process is proposed for the formation of $\mathrm{MgO}$ whiskers. The PL spectrum shows prominent visible emission bands peaked at $2.91 \mathrm{eV}$ in the blue region and $2.39 \mathrm{eV}$ in the blue-green region, extending the potential application of $\mathrm{MgO}$ whiskers in optoelectronic sub-micron devices.

\section{Acknowledgments}

This work was supported by Inha Research Fund 2006.

\section{References}

[1] H.W. Kim, J.W. Lee, J.-H. Yang, Acta Phys. Pol. A 113, 1017 (2008).

[2] F. L. Deepak, P. Saldanha, S.R.C. Vivekchand, A. Govindaraj, Chem. Phys. Lett. 417, 535 (2006).

[3] H. Niu, Q. Yang, K. Tang, Y. Xie, Micropor. Mesopor. Mater. 96, 428 (2006).

[4] J. Zhang, L. Zhang, Chem. Phys. Lett. 363, 293 (2002).

[5] G.H. Rosenblatt, M.W. Rowe, G.P. Williams, Jr., R.T. Williams, Y. Chen, Phys. Rev. B 39, 10309 (1989).

[6] K. Vanheusden, W.L. Warren, C.H. Seager, D.R. Tallant, J.A. Voigt, B.E. Gnade, J. Appl. Phys. 79, 7983 (1996).

[7] J. Guojian, Z. Hanrui, Z. Jiong, R. Meiling, L. Wenlan, W. Fengying, Z. Baolin, J. Mater. Sci. 35, 63 (2000).

[8] S. Schintke, S. Messerli, M. Pivetta, F. Patthey, L. Libioulle, M. Stengel, A.D. Vita, W. Schneider, Phys. Rev. Lett. 87, 276801 (2001). 